\title{
Prevalence of Multidrug Resistant Staphylococcus aureus in Clinically Suspected Atypical Pneumonia Patients
}

\author{
Meenakshi Kante ${ }^{1}$, Rishi Gowtham Racherla ${ }^{2}$, Usha Kalawat ${ }^{3}$, B. Venkata Ramana ${ }^{4}$, D. T. Katyarmal ${ }^{5}$, Abha Chandra $^{6}$, \\ D. Bhargavi , G. Aruna8, Manohar B. ${ }^{9}$
}

\begin{abstract}
${ }^{1}$ Department of Microbiology, Sri Venkateswara Institute of Medical Sciences, Tirupati, Andhra Pradesh, India. ${ }^{2}$ Department of Microbiology, Sri Venkateswara Institute of Medical Sciences, Tirupati, Andhra Pradesh, India. ${ }^{3}$ Department of Microbiology, Sri Venkateswara Institute of Medical Sciences, Tirupati, Andhra Pradesh, India. ${ }^{4}$ Department of Microbiology, Sri Venkateswara Institute of Medical Sciences, Tirupati, Andhra Pradesh, India. ${ }^{5}$ Department of Medicine, Sri Venkateswara Institute of Medical Sciences, Tirupati, Andhra Pradesh, India. ${ }^{6}$ Department of Cardio Thoracic Surgery, Sri Venkateswara Institute of Medical Sciences, Tirupati, Andhra Pradesh, India. ${ }^{7}$ Department of Medical Oncology, Sri Venkateswara Institute of Medical Sciences, Tirupati, Andhra Pradesh, India. ${ }^{8}$ Department of Pulmonary Medicine, Sri Venkateswara Medical College, Tirupati, Andhra Pradesh, India. ${ }^{9}$ Department of Paediatrics, Sri Venkateswara Medical College, Tirupati, Andhra Pradesh, India.
\end{abstract}

\section{ABSTRACT}

\section{BACKGROUND}

Staphylococcus aureus (S. aureus) is one of the common pathogens frequently causing pneumonia. S. aureus is a gram-positive organism, which belongs to the family Micrococcaceae. Generally atypical pneumonia is caused by Mycoplasma pneumoniae, Legionella pneumophila and Chlamydophila pneumoniae. Typical pathogen $S$. aureus is the most frequent and common nosocomial acquired infection. Now-a-days, antibiotic resistant bacterial strains like $S$. aureus have increased in hospital settings. Developing antibiotic resistance is a major problem with $S$. aureus. We wanted to estimate the non atypical pathogens particularly $S$. aureus in clinically suspected atypical pneumonia cases.

\section{METHODS}

250 clinical samples of sputum, broncho alveolar lavage (BAL) and pleural fluid were collected and investigated with Gram staining, culture and antibiotic sensitivity tests.

\section{RESULTS}

Out of a total of 250 cases, S. aureus was isolated in 19 cases. S. aureus showed highest resistance to Ampicillin (15) (6\%), and Penicillin (15) (6\%). S. aureus showed highest sensitivity to Vancomycin 19 (7.6\%) and Tetracycline 19 (7.6\%).

\section{CONCLUSIONS}

Based on clinical symptoms alone, we cannot identify the organism causing the infection. It is important to determine as to whether it is caused by atypical pathogen or typical pathogen. Sometimes co-infections might be the reason. That's the reason why screening is essential for both atypical and typical pathogens. Clinicians must follow the authentication-based guidelines and proper patient management which will help in reducing the severity of the disease. Mainly screening will help clinicians to give proper treatment as well as decrease the drug resistance.

\section{KEY WORDS}

Pneumonia, Nosocomial Acquired Infection, Sputum, Penicillin G, Drug Resistance

\author{
Corresponding Author: \\ Dr. Usha Kalawat, \\ Professor, (Clinical Virology) \\ Department of Microbiology, \\ Sri Venkateswara Institute of Medical \\ Sciences, Tirupati-517507, \\ Andhra Pradesh, India. \\ E-mail: drushakalawat@gmail.com
}

DOI: $10.14260 / \mathrm{jemds} / 2019 / 837$

Financial or Other Competing Interests: Dr. Kalawat reports grants from Sri Venkateswara Institute of Medical Sciences (SVIMS) University, SBAVP, personal fees from Indian Council of Medical Research, New Delhi, outside the submitted work.

How to Cite This Article:

Kante M, Racherla RG, Kalawat U, et al. Prevalence of multidrug resistant staphylococcus aureus in clinically suspected atypical pneumonia patients. J. Evolution Med. Dent. Sci. 2019;8(51): 3863-3867, DOI:
Submission 21-10-2019,

Peer Review 04-12-2019,

Acceptance 10-12-2019,

Published 23-12-2019. 


\section{BACKGROUND}

The word "pneumonia" is derived from the prehistoric Greek word "pneumon" which means "lung," hence the word "pneumonia" becomes "lung disease. Inflammation of lung's parenchyma may be in one or both lungs". Pneumonia is caused by various pathogens like bacteria, viruses, fungi and parasites. Usually Bacterial Pneumonia caused by both typical and atypical organisms. Atypical pneumonia caused by Mycoplasma pneumoniae, Legionella pneumophila and Chlamydophila pneumoniae. Typical pneumonia caused by Klebsiella pneumoniae, Pseudomonas, E. coli, Acinetobacter, Streptococcus pneumoniae, Staphylococcus aureus etc. ${ }^{1} S$. aureus is a common typical pathogen frequently causing pneumonia. $S$. aureus is a gram-positive organism belongs to the family Micrococcaceae, aerobic, catalase and coagulase positive bacteria. Generally, Staphylococcus species are normal inhabitants in human beings. $50 \%$ of $S$. aureus causing pneumonia isolates was turned in to methicillin-resistant $S$. aureus (MRSA), because of lack of proper clinical management.2 Pneumonia caused by $S$. aureus, is one of the severe infections and often associated with clinical difficulties and a high death rate. High mortality rate due to $S$. aureus pneumonia in the pre-antibiotic period, diverse is between $50 \%$ and $90 \%$. In spite of proper antibiotic therapy, the mortality rate is high approximately $30-50 \% .^{3}$

Various virulence factors play an important role in staphylococcal infections and distinctively concerned in the development of pleuropulmonary infections. Several appear to be distinctively, especially in the most severe manifestation of $S$. aureus pneumonia, the haemorrhagic necrotizing phenotype. Enormous polymorphonuclear leukocyte invasion into the lung parenchyma and the development of micro abscesses are distinctive findings of pneumonia caused by $S$. aureus. ${ }^{4}$

WHO identifies antibiotic resistance as a worldwide public health problem as a consequence of its effect on system of health care together with prolonged hospitalisation, higher costs and increased death rate. In USA, approximately 2 million people acquire severe infections caused by bacteria resistant to as a minimum one suggested antibiotic. Staphylococcus aureus pneumonia is a major public health problem by increasing rates of antibiotic resistance due to not so many treatment alternatives. ${ }^{5}$ Several resistant strains can be a source of clinical situations shifting between superficial infections and serious life-threatening infections. ${ }^{6}$ Hence, $S$. aureus should be frequently isolated from the community and Hospital acquired infections. Cell envelope, ribosome and nucleic acids are the targets for antibiotics in Staphylococci. These days antibiotic resistant strains of $S$. aureus raised in hospital settings. Developing antibiotic resistance is a major problem with $S$. aureus. ${ }^{7}$

The present study is to assess non atypical pathogen particularly $S$. aureus in clinically suspected atypical pneumonia cases.

\section{METHODS}

This is a cross sectional study conducted in Department of Microbiology, Sri Venkateswara Institute of Medical Sciences, a tertiary care teaching hospital, Tirupati. Clinically suspected atypical pneumonia cases were selected as per case definition: fever without chills, headache, myalgia and cough without sputum production. $8,9,10$ Total 250 cases of clinically suspected atypical pneumonia were analysed. Institute Ethics Committee approval was obtained before the starting of the study. Clinical samples of Sputum, Bronchoalveolar lavage and pleural fluid were collected as explained by Isa S. Touhali et $\mathrm{al}^{11}$ after obtaining the informed written consent.

Before processing samples; centrifugation was done at $6000 \mathrm{rpm}$ for bronchoalveolar lavage and pleural fluid. Sputum samples were mixed with equal amount of Dithiothreitol (DTT) for lysis of mucus and centrifuged at $13000 \mathrm{rpm}$ for 10 minutes, supernatant fluid was discarded. Pellet was collected and divided into two portions for staining and culture. First portion of pellet was used for gram staining and Z-N staining by standard method. ${ }^{12}$ Gram staining was done for identification of gram-positive organisms and gram-negative organisms. Z-N staining was done to identify acid-fast bacilli.

Second portion was used for culture to isolate typical organisms. A loopful of second portion of pellet was cultured on Nutrient agar, blood agar and MacConkey agar by standard method. ${ }^{13}$ Typical organisms were identified by based on growth, colony characters and biochemical reactions. Antibiotic sensitivity test (Hi media) was performed for each identified organism to know the sensitivity and resistant pattern. $S$. aureus was identified by based on growth, colony characters and biochemical tests.

\section{Statistical Analysis}

Data was recorded, managed and analysed using Microsoft Excel 2007 (Microsoft Corp, USA).

\section{RESULTS}

Of the total of 250 cases, 19 isolates of $S$. aureus were isolated in culture (Table 1). S. aureus showed highest resistance to Ciprofloxacin 11 (57.89\%). No resistance was seen in Linezolid, Netilmicin, Tetracycline, Vancomycin, Levofloxacin (Table 2). S. aureus showed highest sensitivity to Vancomycin $19(100 \%)$ and Tetracycline 19 (100\%). No sensitivity was seen in Nitrofurantoin (Table 2).

\begin{tabular}{|c|c|c|c|}
\hline Total Cases & Organism & Culture Positive & Percentage \\
\hline 250 & S. aureus & 19 & $7.6 \%$ \\
\hline \multicolumn{3}{|c|}{ Table 1. Total Culture Positive Cases of S. aureus } \\
\hline
\end{tabular}

\begin{tabular}{|c|c|c|}
\hline Antibiotics & Sensitive (\%) & Resistant (\%) \\
\hline Amikacin $(30 \mathrm{mcg} / \mathrm{disc})$ & $3(15.79)$ & $1(5.26)$ \\
\hline Ciprofloxacin $(5 \mathrm{mcg} / \mathrm{disc})$ & $7(36.84)$ & $11(57.89)$ \\
\hline Cefoxitin $(30 \mathrm{mcg} / \mathrm{disc})$ & $10(52.63)$ & $8(42.11)$ \\
\hline Clindamycin $(2 \mathrm{mcg} / \mathrm{disc})$ & $14(73.68)$ & $4(21.05)$ \\
\hline Cotrimoxazole $(25 \mathrm{mcg} / \mathrm{disc})$ & $14(73.68)$ & $5(26.32)$ \\
\hline Erythromycin $(15 \mathrm{mcg} / \mathrm{disc})$ & $12(63.16)$ & $7(36.84)$ \\
\hline Nitrofurantoin $(300 \mathrm{mcg})$ & 0 & $1(5.26)$ \\
\hline Gentamicin $(10 \mathrm{mcg} / \mathrm{disc})$ & $12(63.16)$ & $7(36.84)$ \\
\hline Linezolid $(30 \mathrm{mcg} / \mathrm{disc})$ & $16(84.21)$ & 0 \\
\hline Netilmicin $(30 \mathrm{mcg} / \mathrm{disc})$ & $1(5.26)$ & 0 \\
\hline Tetracycline $(30 \mathrm{mcg} / \mathrm{disc})$ & $19(100)$ & 0 \\
\hline Vancomycin $(30 \mathrm{mcg} / \mathrm{disc})$ & $19(100)$ & 0 \\
\hline Levofloxacin $(5 \mathrm{mcg} / \mathrm{disc})$ & $1(5.26)$ & \\
\hline Table 2 . Antibiotic Resistance and Sensitivity Pattern in S. aureus \\
\hline
\end{tabular}




\section{DISCUSSION}

S. aureus is a gram positive typical pathogen causing pneumonia mainly in hospital settings. ${ }^{1} S$. aureus in hospitalized patients with pulmonary infections involved in three main subsets of pneumonia: hospital-acquired pneumonia (HAP), health care associated pneumonia (HCAP) and Ventilator-associated pneumonia (VAP). ${ }^{14}$ HAP- Infection of lung tissue occurs in 48 hours or longer following the hospitalization of a patient with non-intubation. VAP-Hospital acquired infection of lung tissue that generally occurs in 48 hours or longer following intubation for mechanical ventilation. HCAP-Acute infection of lung parenchyma occurring from health care settings includes dialysis centres, nursing homes, Outpatient clinics or hospitalized patients within the past 3 months period. This was previously incorporated in HAP later became a separate group because of some cases appearing as outpatients with pneumonia had been infected by way of multidrug-resistant (MDR) strains previously associated with HAP. Untreated pneumonia can lead to extensive damage of the lung finally impairment of lung function occurs and also mortality rates are raised up to $25 \% .^{1}$

S. aureus is an extremely versatile pathogen in humans, which readily acclimatize to changing environment and attain antibiotic-resistance genes through a number of diverse mechanisms. Recently, World Health Organisation classified global resistance threats and with the organisms of high precedence is $S$. aureus. $S$. aureus is a considerable opportunistic pathogen that inhabits in healthy humans and lead to one of the main causes of bacterial infections like pneumonia in the developed world. ${ }^{15}$

We evaluated patients with suspected atypical pneumonia and reported typical pathogen S. aureus in 19 cases among 250 cases. Ruling out whether a patient is infected with atypical or typical pathogen merely by observing the symptoms is difficult; because co infections by $S$. aureus are common. Therefore, thorough investigations for both typical and atypical pathogens are required.

This study results were almost similar to the study conducted by Shah BA et al, ${ }^{16}$ where $S$. aureus is the second most common pathogen causing pneumonia. Our study results are in discordance with other Indian study Para et al ${ }^{17}$ and also various parts of the country, ${ }^{18-21}$ where $S$. pneumoniae was the most common pathogen causing pneumonia.

S. aureus showed highest resistance to Ciprofloxacin 11 (57.89\%), Cefoxitin 8 (42.11), Erythromycin \& Gentamicin 7 (36.84\%), Cotrimoxazole 5 (26.32\%), Clindamycin 4 (21.05\%), Amikacin and Nitrofurantoin 1 (5.26\%) (Table 2). Highest sensitivity was shown to Vancomycin 19 (100\%) and Tetracycline 19 (100\%) followed by Linezolid 16 (84.21\%), Clindamycin \& Cotrimoxazole 14 (73.68\%), Erythromycin \& Gentamicin 12 (63.16\%), Cefoxitin 8 (42.11\%) Amikacin 3 (15.79\%), Netilmicin and Levofloxacin 1 (5.26\%) (Table 2). Vancomycin was the most effective drug against $S$. aureus which was in concordance with other studies from Turkey and in Europe. ${ }^{22}$

In recent times multi drug resistant $S$. aureus strains are developing in pneumonia cases. Different mechanisms are involved in $S$. aureus antimicrobial resistance (AMR).
Resistance was acquired through basic mechanisms like drug inactivation, enzymatic drug modification, drug efflux, drug binding site modification, displacement of drug and bypass mechanisms concerning acquirement of a new drug-resistant target. Increasing of resistance is also by resistance determinants transformed through transportable genetic elements by way of plasmids, transposons and the staphylococcal cassette genetic material or by mutations in chromosomal genes. ${ }^{23}$

Vancomycin resistance was acquired through conjugation process. Other suggested mechanisms are alterations of cell wall and increased cell wall synthesis that may stop Vancomycin from binding to cell wall which results in continuation of cell wall synthesis. Increase in usage of Vancomycin, directed to emergence of two categories of glycopeptide-resistant $S$. aureus. In the first category Vancomycin intermediate-resistant $S$. aureus (VISA) incessant exposure of glycopeptides lead to thickening of the cell wall and poorly cross-linked cell wall. In the second category Vancomycin-resistant S. aureus (VRSA) - High level of resistance is due to acquisition from Enterococcus species of the VanA operon. ${ }^{24}$

Vancomycin is imperative for management of severe pneumonia infections. ${ }^{25}$ One of the study stated emergence of multidrug resistance strains of $S$. aureus comprising resistant to Vancomycin all over the world in such strains noticeable variations observed in physiology, colony morphology and growth characteristics because of high reductive circumstances with depressed acetate metabolism. ${ }^{26}$ In 1980s, first synthesized Ciprofloxacin belongs to the Fluoroquinolones and extensively used in gram positive organisms. Generally, this drug is helpful in extermination of MRSA. Various parts of the world reported MRSA resistance to Ciprofloxacin. ${ }^{27}$ Neeta D Gade et al.,28 reported highest resistance to Ciprofloxacin which is in concordance with our study. Other studies from India 29,30 they also reported $90 \%$ of resistance to Ciprofloxacin. Mehta AP et al., reported gradual increase in Ciprofloxacin resistance over years, which was $39 \%$ in the year 1922 and steadily raised to $68 \%$ in the year of $1996 .{ }^{31}$ In health care settings, Ciprofloxacin cannot be helpful as a good empirical choice for treating $S$. aureus infections because of its highest resistance. Strains showing resistant to Ciprofloxacin tend to show increased resistance to other antibiotics also.32,33

42.11\% Cefoxitin resistance was observed in the current study. Generally, Cefoxitin resistance denotes that it is Methicillin resistant Staphylococcus aureus (MRSA). ${ }^{34}$ MRSA infections are common in hospital settings. As stated, earlier Ciprofloxacin is commonly administered drug in such infections. ${ }^{27}$ Inappropriate treatment management may also be one of the reasons in developing resistant strains. Hence screening must be needed for pneumonia causing pathogens both typical and atypical along with their antibiogram.

\section{CONCLUSIONS}

Multidrug resistant Staphylococcus aureus can be seen in clinically suspected atypical pneumonia patients. Simply based on clinical symptoms we cannot identify the organism causing the infection. It is important to determine as to whether it is caused by atypical pathogen or typical pathogen. 
Sometimes co-infections might be the reason. That's the reason why screening is essential for both atypical and typical pathogens. Clinicians must follow the authenticationbased guidelines and proper patient management which will help in reducing the severity of the disease. Mainly screening will guide clinicians to give proper treatment as well as decrease the drug resistance. Continuous surveillance is necessary to find out the progression of resistance and mechanisms of resistance. Cautious use of antimicrobials is essential to stop the emergence and spread of resistant microbes.

\section{ACKNOWLEDGEMENT}

Authors acknowledge Sri Venkateswara Institute of Medical Sciences (SVIMS) University, India for providing funds to carry out the work (SBAVP), Indian Council of Medical Research, New Delhi for granting Senior Research Fellowship (SRF), DHR-ICMR-VRDL and Department of Microbiology for providing the lab facility to carry out work. This paper is an extract of a PhD thesis work going to be submitted to SVIMS University, Tirupati, Andhra Pradesh, India.

\section{REFERENCES}

[1] Sattar SBA, Sharma S. Bacterial Pneumonia. [Updated 2019 Feb 11]. In: Stat Pearls. Treasure Island (FL): Stat Pearls Publishing 2019. Accessed on 18.09.2019.

[2] Ragle BE, Karginov VA, Wardenburg BJ. Prevention and treatment of Staphylococcus aureus pneumonia with a beta-cyclodextrin derivative. Antimicrob Agents Chemother 2010;54(1):298-304.

[3] González C, Rubio M, Romero-Vivas J, et al. Staphylococcus aureus bacteremic pneumonia: differences between community and nosocomial acquisition. Int J Infect Dis 2003;7(2):102-8.

[4] Adem PV, Montgomery CP, Husain AN, et al. Staphylococcus aureus sepsis and the WaterhouseFriderichsen syndrome in children. $\mathrm{N}$ Engl J Med 2005;353(12):1245-51.

[5] Aliberti S, Reyes LF, Faverio P, et al. Global initiative for meticillin-resistant Staphylococcus aureus pneumonia (GLIMP): an international, observational cohort study. Lancet Infect Dis 2016;16(12):1364-76. Erratum 2016;16(12):1324.

[6] Murray PR, Rosenthal KS, Pfaller MA. Staphylococcus and related organisms. In: Medical Microbiology. $5^{\text {th }}$ edn. Elsevier Mosby Co, 2005: p. 221-36.

[7] Ioannou CJ, Hanlon GW, Denyer SP. Action of disinfectant quaternary ammonium compounds against Staphylococcus aureus. Antimicrob Agents Chemother 2007;51(1):296-306.

[8] Hoa NQ, Larson M, Chuc KNT, et al. Antibiotics and paediatric acute respiratory infections in rural Vietnam: health-care providers' knowledge, practical competence and reported practice. Trop Med Int Health 2009;14(5):546-55.
[9] Medline plus. U.S.A: atypical pneumonia [updated 2014 Aug 25. cited 2016 July 13]. https://medlineplus.gov/ency/article/000079. Htm.

[10] Medscape. U.S.A: atypical bacterial pneumonia imaging; [updated 2015 Dec 16. cited 2016 Aug 16]. emedicine. medscape. com/article/363083.

[11] Touhali IS, Ibrahim AAF, Dawood HN. Conventional methods for the diagnosis of pneumocystis jiroveci in immunocompromised Iraqi patients. Iraqi J Med Sci 2016;14(1):80-7.

[12] Cillóniz C, Ewig S, Ferrer M, et al. Community-acquired polymicrobial pneumonia in the intensive care unit: aetiology and prognosis. Crit Care 2011;15(5):R209.

[13] de Roux A, Ewig S, García E, et al. Mixed communityacquired pneumonia in hospitalised patients. Eur Respir J 2006;27(4):795-800.

[14] Tong SY, Davis JS, Eichenberger E, et al. Staphylococcus aureus infections: epidemiology, pathophysiology, clinical manifestations and management. Clin Microbiol Rev 2015;28(3):603-61.

[15] Haaber J, Penadés JR, Ingmer H. Transfer of antibiotic resistance in staphylococcus aureus. Trends in Microbiol 2017;25(11):893-905.

[16] Shah BA, Singh G, Naik MA, et al. Bacteriological and clinical profile of Community acquired pneumonia in hospitalized patients. Lung India 2010;27(2):54-7.

[17] Para RA, Fomda BA, Jan RA, et al. Microbial etiology in hospitalized North Indian adults with communityacquired pneumonia. Lung India 2018;35(2):108-15.

[18] Lode HM. Managing community-acquired pneumonia: a European perspective. Respir Med 2007;101(9):186473.

[19] Bartlett JG, Mundy LM. Community-acquired pneumonia. N Engl J Med 1995;333(24):1618-24.

[20] Howard LS, Sillis M, Pasteur MC, et al. Microbiological profile of community-acquired pneumonia in adults over the last 20 years. J Infect 2005:50(2)107-13.

[21] Al-Ghizawi GJ, Al-Sulami AA, Al-Taher SS. Profile of community- and hospital-acquired pneumonia cases admitted to Basra General Hospital, Iraq. East Mediterr Health J 2007;13(2):230-42.

[22] Hanif E, Hassan SA. Evaluation of antibiotic resistance pattern in clinical isolates of Staphylococcus aureus. Pak J Pharm Sci 2019;323(Suppl 3):1219-23.

[23] Foster TJ. Antibiotic resistance in Staphylococcus aureus. Current status and future prospects. FEMS Microbiol Rev 2017;41(3):430-49.

[24] Deyno S, Fekadu S, Astatkie A. Resistance of Staphylococcus aureus to antimicrobial agents in Ethiopia: a meta-analysis. Antimicrob Resist Infect Control 2017;6:85.

[25] McGuinness WA, Malachowa N, DeLeo FR. Vancomycin resistance in Staphylococcus aureus. Yale J Biol Med 2017;90(2):269-81.

[26] Prasad UV, Vasu D, Gowtham RR. Cloning, expression and characterization of NAD Kinase from Staphylococcus aureus involved in the formation of NADP (H): a key molecule in the maintaining of redox status and biofilm formation. Adv Biomed Res 2017;6:97. 
[27] Gilbert MJ, Boscia A, Kobasa WD, et al. Enoxacin compared with vancomycin for the treatment of experimental methicillin-resistant Staphylococcus aureus endocarditis. Antimicrob Agents Chemother 1986;29(3):461-3.

[28] Gade ND, Qazi MS. Fluoroquinolone therapy in staphylococcus aureus infections: Where Do We Stand? J Lab Physicians 2013;5(2):109-12.

[29] Tiwari HK, Sapkota D, Sen MR. High prevalence of multidrug-resistant MRSA in a tertiary care hospital of northern India. Infect Drug Resist 2008;1:57-61.

[30] Pai V, Rao VI, Rao SP. Prevalence and antimicrobial susceptibility pattern of methicillin-resistant Staphylococcus aureus [MRSA] isolates at a tertiary care hospital in Mangalore, South India. J Lab Physicians 2010;2(2):82-4.
[31] Mehta AP, Rodrigues C, Sheth K, et al. Control of methicillin resistant Staphylococcus aureus in a tertiary care centre: a five-year study. J Med Microbiol. 1998;16(1):31-4.

[32] Fernandez CJ, Ackerman VP. In vitro studies of ciprofloxacin and survey of resistance patterns in current isolates. Diagn Microbiol Infect Dis 1990;13(2):79-91.

[33] Tsering DC, Pal R, Kar S. Methicillin-resistant Staphylococcus aureus: prevalence and current susceptibility pattern in Sikkim. J Glob Infect Dis 2011;3(1):9-13.

[34] Fernandes CJ, Fernandes LA, Collignon P. Cefoxitin resistance as a surrogate marker for the detection of methicillin-resistant Staphylococcus aureus. J Antimicrob Chemother 2005;55(4):506-10. 